Dialectologia. Special issue, IX (2021), 7-37.

ISSN: 2013-2247

Received 19 January 2021.

Accepted 23 March 2021.

\title{
PERFILES SOCIOLINGÜÍSTICOS DE LOS INFORMANTES DE LOS ATLAS DIALECTALES CATALANES ${ }^{1}$
}

\author{
Eloi BeLLÉs * \\ Universitat de Barcelona \\ ebelles@ub.edu
}

\begin{abstract}
Este artículo analiza los perfiles sociolingüísticos de los sujetos que se utilizaron en las encuestas lingüísticas para la elaboración de los tres principales atlas dialectales catalanes: el Atlas lingüístic de Catalunya $(A L C)$, el Atlas lingüístico de la Península Ibérica $(A L P I)$ y el Atles lingüístic del domini català $(A L D C)$. Teniendo en cuenta que los atlas tradicionales tomaban a NORMs (non-mobile older rural males) como sujeto favorito, se analiza si se siguieron estos parámetros en cada atlas cuando se eligieron los informantes de las encuestas. Después del análisis de los datos, se muestra que el patrón NORM se siguió, en general, en el $A L D C$ y, parcialmente, en el $A L P I$, pero no se siguió en el $A L C$, que es un trabajo muy pobre metodológicamente.
\end{abstract}

\section{Keywords}

atlas lingüísticos, dialectología catalana, NORM, geolingüística, Atlas lingüístic de Catalunya, Atlas lingüístico de la Península Ibérica, Atles lingüístic del domini català

\footnotetext{
${ }^{1}$ La elaboración de este trabajo se ha beneficiado de un contrato predoctoral FPU 18/00070 del Ministerio de Universidades y forma parte del proyecto de investigación "Variación y cambio lingüístico en catalán: análisis y comparación desde las perspectivas geolingüística y lexicográfica dialectal" (PGC2018-095077-B-C43). Agradezco a Maria-Pilar Perea y a Joan Fontana sus valiosas observaciones. * Departament de Filologia Catalana i Lingüística General, Facultat de Filologia i Comunicació. Gran Via de les Corts Catalanes 585, 08007 Barcelona.
} 


\section{SOCIOLINGUISTIC PROFILES OF THE INFORMANTS OF CATALAN DIALECTAL ATLASES}

\section{Abstract}

This paper analyses the sociolinguistic profile of the informants used in the linguistic surveys for the elaboration of the three main Catalan dialectal atlases: the Altas linguistic de Catalunya $(A L C)$, the Atlas lingüistico de la Península Ibérica $(A L P I)$ and the Atles lingüistic del domini català $(A L D C)$. Taking into account that traditional atlases took NORMs (non-mobile older rural males) as favourite informants, it is analysed if these parameters were followed in each atlas when the informants for the linguistic surveys were chosen. After data analysis and discussion, it is shown that NORM pattern was followed most of the times in the ALDC and, partially, in the ALPI, but was not followed in the $A L C$, that works poorly in a methodological level.

\section{Keywords}

linguistic atlases, Catalan dialectology, NORM, geolinguistics, Atlas lingüistic de Catalunya, Atlas lingüístico de la Península Ibérica, Atles lingüistic del domini català

\section{Introducción}

Desde que Jules Gilliéron publicó el Atlas Linguistique de la France (ALF) (19021910), los atlas lingüísticos han sido, junto con las monografías dialectales, uno de los métodos preferidos para representar los resultados de la dialectología tradicional, a menudo llamada geolingüística. Los estudios dialectales catalanes cuentan con una interesante tradición de cartografía lingüística, desde el temprano Atlas lingüístic de Catalunya $(A L C)$ de Antoni Griera, cuyos trabajos se iniciaron en 1912, hasta el reciente Petit atles lingüístic del domini català $(P A L D C)$ de Joan Veny, de carácter interpretativo, en curso de publicación y que aprovecha los materiales del Atles lingüístic del domini català $(A L D C)$, dirigido por el mismo Veny y Lídia Pons (2001-2018). En medio, se encuentra una rica variedad de atlas regionales o temáticos, como el Atlas linguistique des Pyrénées Orientales (ALPO), de Enric Guiter (1966), el Atlas lingüístic d'Andorra (ALA) de Antoni Griera (1960), el Atles lingüístic de la Terra Alta (ALTA), de Pere Navarro (1996), el Atlas lingüístic de la Diòcesi de Tortosa (ALDT), de Lluís Gimeno Betí (1997), o el Atles lingüístic mariner (ALM) de Francesc de Borja Moll, que aprovecha 
materiales del Atlante linguistico mediterraneo y que el 2018 la Universitat de les Illes Balears publicó en línea.

Desde siempre, uno de los puntos más delicados a la hora de planificar y ejecutar un atlas ha sido su orientación metodológica; en este sentido, son especialmente importantes los criterios para determinar las localidades que deben encuestarse y, sobre todo, el perfil que deben tener los informantes que se someten al interrogatorio lingüístico. Por ejemplo, son sabidas las críticas que recibió durante mucho tiempo el ALC y su autor, Antoni Griera, por el poco cuidado en la elección de las localidades y de sus informantes (Sanchis Guarner 1953, 1955).

Los atlas lingüísticos, por su naturaleza, ofrecen una fotografía de un momento concreto del estado de la lengua en un territorio amplio, por lo que la elección del informante o los informantes de cada localidad es de suma importancia, quizá el aspecto más importante (Chambers \& Trudgill 1998: 29). Y lo es porque el individuo o individuos seleccionados deben representar el habla media de la dicha localidad en una generación concreta, pues no hay ningún atlas románico que haya encuestado sistemáticamente las tres generaciones prototípicas - jóvenes, adultos y ancianos. La coherencia generacional entre los distintos informantes, que parece que no fue muy cuidada por Edmont en los trabajos del $A L F$, cobró importancia - al menos en el plano teórico- desde el AIS de Jud y Jaberg. Ya también desde entonces el perfil clásico de informante que se ha buscado siempre ha sido muy concreto: hombres de edad avanzada, que se hayan movido poco de la localidad, con la ascendencia fijada en ella $-y$, a poder ser, también su cónyuge-, con poca formación y de profesiones agrícolas, lo que ha llevado a denominarlos con el famoso acrónimo NORMs, del inglés non-mobile older rural males, (Chambers \& Trudgill 1998: 29).

Non-mobile para no estar influido por el habla de otras localidades; older por conservar, los ancianos, un lenguaje más "puro", más cercano al ideal de lengua no corrompida que caracterizaba el objeto de la primera geolingüística; rural porque la mayor parte de los cuestionarios estaban repletos de términos, acciones y preguntas relativas a la vida del campo y el modo de vida tradicional que con el progreso, poco a poco, iba pereciendo; y males porque, según justificaban los propios encuestadores, 
conocían más la vida rural y tenían más disponibilidad que las mujeres, además de ocultar un cierto machismo. ${ }^{2}$ Asimismo, cabe añadir que a menudo se buscaban individuos con poca o nula formación académica, de modo que la lengua estándar o literaria no influyera sobre su dialecto.

El objetivo de este trabajo es analizar estas variables en el perfil sociolingüístico de los informantes de los atlas catalanes. ${ }^{3}$ Se han considerado solamente los tres atlas que tienen una visión total del dominio lingüístico, es decir, el $A L C$, el $A L D C$ y el Atlas lingüístico de la Península Ibérica $(A L P I)$, en el que, aunque el dominio catalán sea solo una parte del proyecto, es completo y autónomo, y contó con un equipo propio de encuestadores. No se han incluido, por tanto, ni los atlas parciales ni tampoco el PALDC, que usa los mismos datos $-\mathrm{y}$, pues, los mismos informantes- que el ALDC.

Para llevar a cabo el estudio se han sistematizado las informaciones sobre los sujetos que aparecen en las páginas preliminares del ALC (Griera 1923: s. p.; 1964) y del $A L D C$ (I, 33-95); ${ }^{4}$ para el $A L P I$ se han obtenido directamente de la base de datos de los trabajos en curso de digitalización del CSIC. Las informaciones que se dan de cada sujeto, como es obvio, no son iguales en los tres atlas, ni tampoco son exhaustivas. En efecto, cada atlas elaboraba la ficha de los sujetos de la encuesta de un modo diverso. Sin embargo, hay elementos comunes en todas las encuestas: el nombre del sujeto que, careciendo de interés sociolingüístico, se ha omitido-, la edad, el sexo, el arraigo en la localidad, el oficio, y, generalmente, el nivel de instrucción.

El presente trabajo se divide en cuatro partes, sin contar esta introducción. En las tres primeras, se analiza cada atlas de forma individual, siguiendo siempre el mismo patrón: una breve descripción de la obra seguida de un análisis de los datos que ofrece

\footnotetext{
2 "Como en el cuestionario del ALPI predomina la terminología agrícola, en general hemos preferido interrogar a los hombres y no a las mujeres, que, en muchas regiones, apenas intervienen en las labores del campo; por otra parte, las mujeres solían tardar más a hacerse cargo de la índole de nuestra labor, y además, por presunción, siempre tienden más que los hombres a refinar su habla vulgar ante el forastero; aparte de ello, mientras que en determinadas épocas del año es relativamente fácil contratar a los campesinos poco ocupados para las varias jornadas que durará el interrogatorio, las mujeres nunca se hallan libres de sus labores domésticas y se niegan a comprometerse para la totalidad de la encuesta" (Sanchis Guarner 1953: 57).

${ }^{3}$ Hay algunos precedentes que han analizado comparativamente la metodología de los atlas catalanes, como por ejemplo Sanchis Guarner (1955), Gargallo (1990) o españoles en su totalidad, como González González (1992). Son trabajos que, sin embargo, no se centran en el perfil de los informantes sino más bien en el enfoque metodológico en su conjunto.

${ }^{4}$ Información que se reproduce, también, en la versión web.
} 
sobre sus informantes. Se presenta por orden cronológico de la elaboración de las encuestas y el inicio de su publicación, y así en primer lugar se encuentra el Atlas lingüístic de Catalunya, seguido del Atlas lingüístico de la Península Ibérica y, finalmente, el Atles lingüístic del domini català. En la cuarta parte se ofrece un comentario comparado de los resultados de los tres análisis previos y se presentan las conclusiones.

\section{El Atlas lingüístic de Catalunya, de Antoni Griera}

El Atlas lingüístic de Catalunya $(A L C)$ tiene el mérito de ser el primer atlas publicado en la Península Ibérica, y también de ser uno de los primeros fuera de la órbita del francés y sus variedades. Aun así, las graves deficiencias metodológicas y de elaboración de los materiales que se han señalado unánimemente han cuestionado -quizá sería más apropiado decir invalidado- la utilidad del atlas. De hecho, es sabido que su autor, Antoni Griera, cuidaba poco los aspectos metodológicos y la rigurosidad tanto en los resultados como en las hipótesis lingüísticas que formulaba. ${ }^{5}$

El proyecto para disponer de un atlas lingüístico del catalán empezó muy tempranamente, y casi coincidió con la finalización del ALF de Jules Gilliéron, considerado el padre de la geolingüística $-\mathrm{y}$ de hecho coincidió también con los planteamientos iniciales del Atlas lingüístico de la Península Ibérica (cfr. § 3). El 1907 la Diputación de Barcelona, con el apoyo y el entusiasmo de Bernhard Schädel y Antoni M. Alcover, y alentados por el éxito del I Congreso Internacional de la Lengua Catalana de 1906, decidieron becar tres estudiantes para que estudiaran filología y lingüística románicas en Europa. Los becados fueron Manuel de Montoliu, Pere Barnils y Antoni Griera (Julià-Muné 2019: 73).

Fue este último quien, tras estudiar en París con Gilliéron en el curso 1911-1912, planeó un atlas para que fuese "una extensió de l'Altas Linguistique de la France"

\footnotetext{
${ }^{5}$ No solo en el ALC, también en otras obras, como por ejemplo en el ALA -véase la reseña de Germà Colón (1961), traducida al catalán en Colón (1997)- o en el Vocabulario vasco que publicó en 1960 -duramente reseñado y criticado por Koldo Mitxelena (Michelena 1960; reproducido en Michelena 1985).
} 
(Griera 1923: s. p.; Griera 1964: 5). Con el soporte de la Sección Filológica del Institut d’Estudis Catalans, y de su presidente, Antoni M. Alcover, en 1912 Griera adaptó al catalán el cuestionario que había servido a Gilliéron para la elaboración del Atlas linguistique de la Corse (1911-1914) y realizó las encuestas entre 1912 y 1922. A partir de un momento indeterminado, Antoni M. Alcover - a instancias del propio Griera, según parece- colaboró también en las tareas de encuesta dialectal. En verano de 1917 Alcover participaba en las encuestas de Baleares, y la negativa de Griera -o de Pompeu Fabra - ante la demanda de más cuestionarios aceleró el choque de Alcover con el Institut y su salida. Alcover fue relevado de sus cargos en la Sección Filológica con una durísima carta de su secretario, Josep Carner (Perea 2008: caja 025, documento 25c), a la que siguió el primero de los manifiestos que el manacorense publicó en su Bolletí del diccionari de la llengua catalana (BDLC) contra el Institut (v. Perea 2020a), en el que manifiesta algunas de las discrepancias metodológicas con Griera. Alcover, ya entrenado en la encuesta dialectal en sus múltiples eixides filològiques ('excursiones filológicas'), proponía el uso de más de un informante para cada localidad, así como la participación de más de un encuestador, y el uso de dibujos para ayudar a los informantes a identificar lo que se les pedía. Por su lado, Griera se mantenía fiel a los principios que habían guiado a Gilliéron y que son la base de la geolingüística clásica: un solo informante para cada localidad, un solo encuestador y uso del sistema directo de encuesta, sin dibujos. Además, indica Alcover, discrepaban sobre el sistema de transcripción que se tenía que adoptar - se pueden leer los manifiestos en Alcover (2020, esp. 67-79), en los que incluye las aprobaciones de Pier Enea Guarnerio y de Jakob Jud a sus propuestas metodológicas; la visión de Griera se puede obtener de sus memorias, claramente parciales y sesgadas (Griera 1963: 98100).

Liberado de Alcover, Griera prosiguió con los trabajos del ALC y en 1923 publicó el primer volumen, que presentaba 187 mapas ordenados, como en el $A L F$, por orden alfabético. El $A L C$ continuó publicándose, con algún problema derivado del alejamiento progresivo de Griera y el Institut d'Estudis Catalans, hasta 1939 -tomo V, estriparfregar la roba. Después, los materiales inéditos se perdieron y se daba por muerto el 
proyecto, ${ }^{6}$ hasta que en 1959 Griera convenció a Antoni Pladevall, un joven sacerdote e historiador, para que repitiera en los mismos puntos de encuesta las partes perdidas del cuestionario. La segunda parte del ALC se publicó hasta 1964, con unos materiales, obviamente, que no permitían la colación con aquellos recogidos entre 1912 y 1922 y publicados antes de la guerra. Cuando apareció esta segunda parte ya se habían iniciado los trabajos para elaborar el $A L D C$ y se había publicado el primer volumen del $A L P I$, y el $A L C$ era un proyecto muerto para la comunidad lingüística catalana. En este sentido, y asumiendo la propuesta de Julià-Muné (2019: 78) que considera solo los trabajos del $A L C$ hasta 1939, no se examina la segunda parte - por otro lado, muy deficiente metodológicamente (Sanchis Guarner 1953: 653; Veny 1986: 95).

\subsection{Perfiles sociolingüísticos de los informantes del ALC}

De una obra que se define a sí misma como continuadora de la de Gilliéron se esperaría encontrar unos informantes que perteneciesen, enteramente, a los parámetros clásicos de la geolingüística, es decir, que fuesen hombres ancianos, que se hayan movido poco del pueblo y con poca formación. Igualmente se esperaría encuestar lugares pequeños $y$, pues, conservadores desde un punto de vista lingüístico. La realidad, sin embargo, es muy distinta, y ya Sanchis Guarner (1955: 652653) denunció el poco cuidado de Griera en la elección de los sujetos.

La introducción del primer volumen del ALC (Griera 1923) y la introducción que, en forma de separata, se publicó al final de la obra (Griera 1964), difieren en algunos puntos, como en la presentación del proyecto, su historia y otros detalles menores debidos al cambio de posicionamiento político del autor; ${ }^{7}$ en la segunda introducción se incluye, además, la información de los sujetos con que se completaron las encuestas. En cuanto a los criterios de selección de informantes, los cambios son mínimos. El $A L C$ es el atlas que da menos información sobre sus informantes. Según

\footnotetext{
${ }^{6}$ Por ejemplo, en Badía \& Colón (1952, 1955) o en Sanchis Guarner (1953).

${ }^{7}$ Por ejemplo, en 1923 Griera justificó haber excluido Mahón de la lista de localidades "perquè és una ciutat d'al-luvió, formada de romanalles castellanes de l'excèrcit d'Espanya" (Griera 1923: 1c), mientras que en 1964 la argumentación cambia a "perquè és una ciutat d'alluvió (sic), formada de romanalles sense valor i d'època recent” (Griera 1964: 10).
} 
Griera sería ideal conseguir tres sujetos de cada localidad, uno que representase a la generación anciana, otro a la generación de mediana edad y, por último, a la generación joven. ${ }^{8}$ No obstante, y ante la dificultad que supone encontrar informantes válidos, opta finalmente por el modelo de sujeto único. Este sujeto único debe cumplir cuatro características, según Griera: ser hijo de la localidad encuestada, y no haber vivido en otros lugares; ser un sujeto de edad avanzada; ser inteligente e ilustrado, y ser hombre - solo incluye una mujer, en la localidad de Sallagosa (Alta Cerdaña).

Aun así, Griera reconoce que no siempre se ha conseguido encontrar un sujeto válido, y advierte de que los informantes de Valencia son, a menudo, "peores" que los de Cataluña y Baleares. De las cuatro características teóricas que establece Griera, tres encajan a la perfección en el patrón clásico de NORM: hombres de edad, del lugar y sin viajes. Sorprende la preferencia por sujetos ilustrados, que justamente con el hecho de que la mayor parte de las localidades sean grandes núcleos de población, es decir, no rurales, rompe los esquemas clásicos. ${ }^{9}$ Sin embargo, que la propuesta de Griera encajase teóricamente en el patrón NORM no implicó que los sujetos finales cumpliesen estas características. A continuación, se resumen, en forma de tabla, los perfiles sociolingüísticos de los 110 informantes del primer período de encuestas en las $101^{10}$ localidades totales. ${ }^{11}$

\footnotetext{
${ }^{8}$ Que es, en el fondo, el planteamiento de Alcover contra el que se rebeló Griera.

${ }^{9}$ Griera justifica el hecho de encuestar grandes ciudades por ser el núcleo de irradiación de los cambios lingüísticos.

${ }^{10}$ Según Alcover (2020: 541), tenían que ser 250, pero los cambios en el planteamiento de la obra redujeron el número final. Son muchos quienes han criticado la ausencia de más localidades valencianas y el exceso de puntos de encuesta del catalán oriental y occidental, como Sanchis Guarner (1955), Veny (1986: 74, 90).

${ }^{11}$ En Santa Coloma de Queralt, Vilafranca del Penedès, Morella, Alcira, Llucmajor, Ciutadella y el Alguer hay más de un informante.
} 


\begin{tabular}{|c|c|c|c|c|c|c|c|}
\hline \multirow{2}{*}{ Sexo } & \multicolumn{2}{|c|}{ Hombres } & \multicolumn{2}{|c|}{ Mujeres } & \multicolumn{2}{|c|}{ Indeterminado $^{12}$} & Total \\
\hline & \multicolumn{2}{|l|}{107} & \multicolumn{2}{|l|}{2} & \multicolumn{2}{|l|}{1} & 110 \\
\hline \multirow{2}{*}{ Ocupación } & \multicolumn{2}{|c|}{ Agrícola ${ }^{13}$} & \multicolumn{2}{|c|}{ Industrial $^{14}$} & \multicolumn{2}{|c|}{ Intelectual $^{15}$} & \multirow{2}{*}{87} \\
\hline & \multicolumn{2}{|l|}{37} & \multicolumn{2}{|l|}{11} & \multicolumn{2}{|l|}{39} & \\
\hline \multirow{2}{*}{ Edad } & Hasta 30 & $31-40$ & $41-50$ & $51-60$ & $61-70$ & 71 o más ${ }^{16}$ & \multirow{2}{*}{101} \\
\hline & 30 & 12 & 18 & 17 & 15 & 9 & \\
\hline
\end{tabular}

Tabla 1. Datos de sexo, ocupación y edad de los informantes del ALC.

A partir de los datos que Griera ofrece, se constata que solo se cumple casi sistemáticamente un criterio, el de la masculinidad del sujeto. En efecto, de las dos mujeres encuestadas, solo una (Sallagosa) es sujeto único. En Vilafranca del Penedès es la mujer del informante principal $y$, por último, un caso indeterminado en Llucmajor, que no se puede resolver.

Un breve repaso a la ocupación - que es el único modo de intuir el nivel de formación de los sujetos, una de las variables consideradas por Griera- indica claramente que una parte nada menospreciable de los informantes cumplía su criterio, eso es, haber recibido una formación reglada. De entre los 39 informantes que se han incluido en el epígrafe "Intelectual", destaca la gran presencia de eclesiásticos - hasta 16 curas $-y$ de estudiantes -11 en total. Esto en parte se explica porque el propio Griera era sacerdote, y le resultaba enormemente fácil llegar a una aldea y, sin buscar otro sujeto, encuestar a los eclesiásticos del lugar o bien a jóvenes estudiantes o seminaristas. Algunas de estas encuestas, de hecho, no se realizaron in situ, sinó que Griera aprovechó viajes de sus informantes a Barcelona, donde él residía, para llevar a cabo la investigación. Es el caso de Durro, Sant Hilari Sacalm o Morella.

\footnotetext{
${ }^{12}$ Un(a) informante de Llucmajor, de profesión indefinida, se llama "Lluc", que generalmente es nombre masculino pero que en Mallorca es, casi siempre, nombre de mujer en honor al santuario de Maria de Lluc. Ante la duda, prefiero no asignarle sexo.

${ }^{13}$ Incluye los epígrafes "pagès", "espardenyer i pagès", "agricultor", "hortolà", "pagès i mariner", "llaurador", "conrador", "mariner", "masover", "missatge", "propietari rural” y "esposa de l'anterior" (esposa de un sujeto que es "propietari rural").

${ }^{14}$ Incluye los epígrafes "comerciant", "ferrer", "mestre de cases", "barber", "carreter", "agutzil" y "fuster".

${ }^{15}$ Incluye los epígrafes "capellà", "estudiant", "mestre", "mestre de minyons", "advocat i propietari", "notari", "metge", "impressor", "filòleg", "lingüista" y "arxiver".

${ }^{16}$ Se incluyen aquí dos sujetos cuya edad es, solamente, "més de 60 anys".
} 
Comentario a parte merecen los informantes de Centelles, Barcelona, Cocentaina, Palma y el Alguer. Los informantes de estos lugares fueron, respectivamente, el lingüista Pere Barnils, el filólogo Pompeu Fabra, el lingüista Lluís Fullana, el escritor Bartomeu Ferrà y el gramático Joan Palomba. ${ }^{17}$ El caso más extremo es, sin duda, el sujeto de Sant Bartomeu del Grau, cuyo informante fue el mismo Antoni Griera. Si entre los primeros dialectólogos el debate era acerca de la necesidad de que el investigador fuese o no lingüista, puesto que su conocimiento de la realidad dialectal podía influir en la percepción de la respuesta, el hecho de que lo fuese el propio sujeto desacredita, totalmente, estas encuestas.

En cuanto a la edad de los informantes, también se ve claramente que Griera no consiguió -o no quiso conseguir - sujetos mayormente ancianos, como se proponía. La franja de edad con más informadores es, justamente, la de la población más joven. Esto se explica, sin duda, por la gran presencia de estudiantes, como ya se ha visto. Todos los sujetos de más de 60 años suman solo 21 individuos, apenas una quinta parte del total, hecho que no deja de sorprender si se tiene en cuenta que se buscaban individuos ancianos. La gran disparidad de edad entre los sujetos también rompe otro de los principios básicos de la geolingüística tradicional: no quebrantar la unidad generacional entre las diversas encuestas.

No queda sino analizar el primer criterio, es decir, que el sujeto sea natural de la localidad y apenas haya viajado. Griera no da información sistemática sobre este aspecto, pero en los comentarios que incluye en la descripción de cada encuesta pueden hallarse algunas indicaciones. En total, hasta 20 informantes han pasado temporadas más o menos largas fuera de su localidad - algunos, incluso, no viven allí, solo van en vacaciones-, sin contar que Griera y Barnils habían estudiado juntos en Alemania, o que Fabra acababa de regresar de Bilbao tras 10 años de vivir allí. Además, hay dos informantes nacidos fuera de la localidad. En total, pues, una veintena larga de sujetos no cumplen con el requisito de la inmovilidad en la localidad de origen, muchos de los cuales debido a los estudios.

\footnotetext{
${ }^{17}$ Y aún se podría considerar incluir en esta lista los informantes de Binèfar, un abogado que declara haber escrito un vocabulario de la localidad, y de Gandesa, un labrador que publicó unas Poesies populars gandesanes.
} 
Para terminar, las observaciones de Griera dan aun algunas informaciones interesantes sobre aspectos diversos, como la competencia en el dialecto de la localidad, la ausencia de dientes (Benasc), etc.; quizá es llamativo el caso de Tamarit de la Llitera, cuyo informante es un noble que declara ser de familia bilingüe -otro quebrantamiento del modelo clásico NORM.

Se demuestra, pues, el poco rigor metodológico - incluso en la metodología que Griera mismo se marcó- en la recogida inicial de los materiales del ALC. ¿No había ancianos disponibles en ninguna de las localidades en las que toma estudiantes? Capitales de provincia, de obispado, de partido judicial, ¿no tenían variedad de sujetos entre los que elegir? ¿O más bien fue Griera que buscaba una solución fácil para su tarea? Los serios reparos que se han señalado hacia el ALC se confirman, también, en cuanto a los sujetos.

\section{El Atlas lingüístico de la Península Ibérica, dirigido por Tomás Navarro Tomás}

La idea de elaborar un atlas lingüístico "nacional” para toda España, a imagen del Atlas lingüistique de la France, nació casi a la par que el proyecto del ALC. Sin embargo, mientras que los trabajos para aquel comenzaron rápidamente, los trabajos de este se demoraron por más tiempo. En efecto, como cuenta el director del atlas Tomás Navarro Tomás, justo después de la publicación del ALF Ramón Menéndez Pidal tuvo la idea de preparar un atlas similar, cuya dirección finalmente recaería sobre Navarro Tomás. En 1914 el alcance del atlas ya estaba definido -todos los romances ibéricos-, así como su nombre, Atlas lingüístico de la Península Ibérica (ALPI). El objetivo era ofrecer una panorámica del continuum románico de la península, formado por los bloques galaicoportugués, castellano y catalán, respectivamente (Navarro Tomás 1975). ${ }^{18}$

El cuestionario, común para todas las localidades, fue elaborado por Navarro Tomás a partir del cuestionario del AIS, y supervisado por Menéndez Pidal. Se dividía en dos cuadernos, uno para la fonética - más breve- y otro para el léxico y la

\footnotetext{
18 Para una historia interna del ALPI puede consultarse Cortés Carreres \& García Perales (2009).
} 
etnografía -más amplio. Las encuestas fueron a cargo de grupos de dos investigadores naturales de cada ámbito geográfico, especialmente formados por Navarro Tomás. Para el ámbito catalán se ocuparon de las encuestas Manuel Sanchis Guarner (fonética) y Francesc de Borja Moll (léxico). La mayor parte de las encuestas tuvieron lugar durante la década de 1930, pero la Guerra Civil interrumpió los trabajos, que se terminaron entre 1947 y 1952 . A menudo se ha reparado en la diferencia de resultados como consecuencia del corte cronológico, y ya Colón \& Lütdke (1965) señalaron que esta disparidad podría afectar a los resultados finales. Tras muchas penurias económicas, el 1962 se publicó el primer -y único-volumen de fonética. A partir de 2009 un equipo de investigadores, dirigido por Pilar García Mouton, ha reanudado la edición digital de los materiales del $A L P I$, aún en proceso de publicación. ${ }^{19}$

Las informaciones sobre los sujetos del ALPI ya forman parte de la base de datos del CSIC para su publicación, pero todavía son inéditas, pues en el volumen de 1962 se decía que se detallarían en un anejo nunca publicado ( $A L P I, I, 9-10)$. Sin embargo, en diversos trabajos Navarro Tomás y Sanchis Guarner, entre otros, hablaron de los planteamientos metodológicos del $A L P I$, por lo que es sabida la teoría sobre el perfil de los sujetos. Así, en la noticia histórica del ALPI que dio Navarro Tomás se decía que el objetivo era "ofrecer una representación de la lengua popular hablada en pueblos menores y antiguos por personas iletradas o de escasa cultura, entre los cuarenta y los sesenta años de edad" (Navarro Tomás 1975: 9).

También exponía que para la parte de fonética se había tomado un único informador, mientras que para la parte etnográfica y de léxico se habían servido de varios sujetos según las necesidades de la materia (Navarro Tomás 1975: 14). En diversos lugares así lo había explicitado Sanchis Guarner - solo $(1953,1955)$ o con otros colegas (Sanchis Guarner et al. 1962). En síntesis, para el ALPI se prefirieron los pueblos pequeños a los grandes, y se buscaron sujetos que, como es lógico, "hablasen bien el dialecto" (Sanchis Guarner 1953: 57). Para el lingüista valenciano, "no solo han de ser naturales los sujetos de la localidad estudiada, sino que también debe

19 La información del proyecto y los resultados preliminares se pueden consultar en http://www.alpi.csic.es [consulta: 18.1.2021]. 
procurarse que lo sean sus padres y sus esposas. Se recomienda que hayan viajado poco y también que sean analfabetos o poco instruidos, a fin de que se vean exentos de la influencia de los dialectos de otras localidades y de la lengua culta" (Sanchis Guarner 1953: 57).

Además, se prefieren los hombres a las mujeres, puesto que las mujeres conocen menos las tareas agrícolas, y a menudo tienen menos disponibilidad para dejar sus tareas (Sanchis Guarner 1953: 57). Por lo que a la edad se refiere, sigue Sanchis, "hemos preferido generalmente sujetos de edad madura, cuya habla es, naturalmente, más arcaizante y está menos influida [...] pero hemos evitado los demasiado viejos" (1953: 57-58); es, en suma, lo que manifiestan Sanchis et al. (1962: 116) cuando explican que

las investigaciones dialectales se realizaban normalmente interrogando a sujetos rústicos, analfabetos o poco instruidos, de edad madura y que hubiesen viajado poco, a fin de que se viesen exentos de la influencia de la lengua culta y de los dialectos de otras localidades.

Como en el cuestionario del ALPI predomina la terminología agrícola, por lo regular fueron interrogados hombres y no mujeres, ya que éstas en muchas regiones apenas intervienen en las labores del campo.

En síntesis, pues, se ve claramente como los parámetros en que se concibió y se explicó el ALPI son claramente coincidentes con la geolingüística tradicional y, por lo que concierne a los informantes, con el perfil NORM. Sin embargo, el análisis de los datos hará matizar esta afirmación.

\subsection{Perfiles sociolingüísticos de los informantes del ALPI}

Como se ha dicho, las informaciones sobre los sujetos del ALPI no están aún publicadas. Sin embargo, el hecho de que hayan sido vaciadas en la base de datos del CSIC ha permitido recuperarlas y analizarlas en este trabajo. En la edición actual del $A L P I$ se ha asignado al equipo catalán, dirigido por Maria-Pilar Perea, un total de 111 
localidades; de estas, 15 no pertenecen al dominio lingüístico catalán. ${ }^{20}$ Todas fueron investigadas entre 1934 y 1952 por Manuel Sanchis Guarner, acompañado de Francesc de B. Moll en las del dominio lingüístico catalán y de Lorenzo Rodríguez Castellano en las de lengua castellana. Las localidades castellanohablantes de Monforte y Dolores (Alicante) fueron investigadas previamente, en 1932, por Aurelio M. Espinosa hijo y Lorenzo Rodríguez Castellano. ${ }^{21}$ Como parece que cada equipo de encuestadores tenía un patrón propio para elegir a los informantes (García Mouton 2020), estas dos localidades no se han tenido en cuenta.

El cuestionario del ALPI preveía las siguientes informaciones para el sujeto o informador: sexo, edad, localidad natal, ¿sabe leer?, naturaleza del padre, de la madre, viajes y otros. A menudo los investigadores añaden información acerca del grado de conocimiento del castellano -o del valenciano en los territorios castellanohablantes de Valencia.

De los 111 puntos de encuesta, en Anna (cuatro informantes), Teresa de Begís (dos informantes) y Godelleta (dos informantes) se opta por más de un sujeto en el cuaderno I (fonética). En los otros casos, se usa el sujeto único en esta parte. En el cuaderno II hay sujetos múltiples en Taurinyà (dos informantes), Godelleta (dos informantes), Teresa de Begís (dos informantes) y Cornellà de Llobregat (tres informantes). En las demás localidades se sirven de un solo informante. Además, en Llo, Arles de Tec, València d’Àneu, el Pont de Suert, Rialb, Seterada, Alàs, Organyà, Bassella, Bolvir, Agullana, Viladecavalls, Godelleta y Beniopa no hay datos del informante del cuaderno II, y en Anna el informante del cuaderno II coincide con el primero del cuaderno I, aunque son encuestas hechas en distintos años. ${ }^{22}$ En Rafaelbunyol solo hay datos del sujeto del cuaderno II. En Capdepera falta el cuaderno II, que seguramente se perdió. En total, pues, se ha trabajado sobre una base de 112 informantes en los cuadernos de fonética y 95 para el cuaderno de léxico, que suman 207 informantes.

\footnotetext{
${ }^{20}$ Son Castillo de Villamalefa, Fanzara, Teresa de Begís, Azuébar, Ademuz, Tuéjar, Marinas, Caudete de las Fuentes, Pedralba, Godelleta, Dos-aguas, Teresa de Cofrentes, Anna, Monforte y Dolores.

${ }^{21}$ El mapa no 5 de la edición del ALPI de 1962 informa del equipo de encuestadores de cada localidad.

22 El cuaderno I se encuestó en 1948, el cuaderno II, en 1934; solo se tienen en cuenta los datos del cuaderno II, más antiguo, porque es cuando se consideró que era un informante óptimo.
} 
En cuanto al sexo, se observa como efectivamente los sujetos femeninos son prácticamente anecdóticos: solo cuatro mujeres de 210 encuestados, y todas en el cuaderno de fonética - hay que recordar que se consideraban poco aptas por su bajo conocimiento del mundo rural, temática que se desarrollaba en el cuaderno II. Sin embargo, vale la pena remarcar que en otros puntos del $A L P I$ se observan más mujeres entre los informantes, especialmente entre las encuestas que hicieron Lorenzo Rodríguez Castellano y Aurelio M. Espinosa (García Mouton 2020).

\begin{tabular}{|c|c|c|c|}
\hline \multirow{2}{*}{ Sexo } & Hombres & Mujeres & Total \\
\cline { 2 - 4 } & 203 & 4 & 207 \\
\hline
\end{tabular}

Tabla 2. Sexo de los informantes del ALPI.

En la edad de los sujetos se observa una tendencia a la madurez, aunque no a la senectud. No hay, a diferencia del $A L C$ y el $A L D C$, ningún informante menor de 30 años, y muy pocos que pasen de los 70, por lo que la homogeneización de los datos sería mayor en este atlas si las encuestas no hubiesen padecido el corte de la guerra. Sin embargo, teniendo en cuenta la gran cantidad de informantes de mediana edad -la mitad tienen menos de 60 años - es difícil considerar que el $A L P I$ sea un paradigma de NORM, al menos por lo que a la edad se refiere, aunque como se ha visto sus promotores así lo manifestasen.

\begin{tabular}{|c|c|c|c|c|c|c|c|}
\hline \multirow{2}{*}{ Edad } & 40 o menos & $41-50$ & $51-60$ & $61-70$ & $71-80$ & 81 o más & Total \\
\cline { 2 - 8 } & 5 & 40 & 53 & 81 & 24 & 4 & 207 \\
\hline
\end{tabular}

Tabla 3. Edad de los informantes del ALPI.

En cuanto a la naturaleza de los informantes, sí que se siguió el planteamiento inicial de utilizar solo individuos naturales del lugar. En efecto, de 210 informantes, solo ocho no son naturales del lugar, ${ }^{23}$ y aún en algunos casos Sanchis y Moll justifican

\footnotetext{
${ }^{23}$ Clariana (cuaderno I), Molló (cuaderno I), La Valldan (cuaderno I), Dolores (cuaderno I), Mont-ras (cuaderno II), Sant Martí de Sesgueioles (cuaderno II), Cornellà (cuaderno II), Maó (cuaderno II).
} 
debidamente el hecho. ${ }^{24}$ No pudieron ser tan estrictos en la ascendencia de los informantes ${ }^{25} y$, todavía menos, en la falta de movilidad. Sin embargo, sí que parece sólido considerar que en este aspecto el patrón NORM fue respetado en el ALPI.

\begin{tabular}{|l|c|c|c|}
\cline { 2 - 3 } \multicolumn{1}{c|}{} & Sí & No & Total \\
\hline Natural de la localidad & 200 & 7 & 207 \\
\hline Padre de la localidad & 162 & 28 & 190 \\
\hline Madre de la localidad & 145 & 45 & 190 \\
\hline Ambos progenitores de la localidad & 177 & & \multicolumn{1}{|c|}{} \\
\hline Sin ninguna ascendencia en la localidad & & 13 & \multirow{2}{*}{$202^{26}$} \\
\hline Ha vivido fuera (más de seis meses) & 97 & 105 & \multicolumn{2}{|c|}{} \\
\cline { 1 - 3 }
\end{tabular}

Tabla 4. Naturaleza, ascendencia y movilidad de los informantes del ALPI.

Se ve claramente como se respetó el principio de naturaleza del sujeto, y se ve también que se buscó que sus progenitores fueran de la misma localidad. Un gran número de ellos cumplen con los requisitos, y muy pocos no tienen ninguna ascendencia en la localidad encuestada. Los principios teóricos que guiaron el $A L P I-\mathrm{y}$ la geolingüística tradicional- se cumplen de un modo general. En cambio, el anhelo de encontrar informantes que a penas hubiesen viajado resultó más difícil, en vista de los resultados. La mayor parte de los desplazamientos se deben al servicio militar obligatorio o a motivos bélicos (muchos en Cuba, Filipinas y el África); pero también hay motivos económicos: emigraciones temporales a Francia, a otros lugares de España, a América, etc.

Visto que el cuestionario etnográfico-léxico era eminentemente rural y agrícola,

\footnotetext{
${ }^{24}$ Es el caso por ejemplo de La Valldan, cuyo informante nació a $6 \mathrm{~km}$ del pueblo y se trasladó allí a los seis meses; de Clariana de Cardener, cuyo informante era nacido accidentalmente en el municipio limítrofe, pero en una masía diseminada, puesto que allí no hay núcleos de población; y de Molló, de cuyo informante Sanchis dice: "Hace treinta años que vive en Molló y antes había residido siempre en Surroca o en Sant Joan de les Abadeses; todos los pueblos citados son de la comarca del Ripollés pero Molló es más montañoso. No supimos que el sujeto no era de Molló hasta que ya teníamos el cuaderno casi acabado; no hay grandes diferencias de habla".

${ }^{25}$ No hay datos de los informantes del cuaderno I de Anna (en tres de los cuatro), Benilloba ni Maó (del que se dice que era huérfano), ni del cuaderno II en Taurinyà (dos informantes), Bell-lloc d'Urgell, Clariana de Cardener, Besalú, Anglès, Sant Martí Sesgueioles, Cornellà de Llobregat (dos informantes), Sant Joan d'Eivissa y Eivissa.

${ }^{26}$ Sin datos de tres informantes de Anna (cuaderno I) ni de los de Bell-lloc d'Urgell y Guardamar (cuaderno II).
} 
se buscaban informantes que se ocupasen profesionalmente en este ámbito. Como se puede ver en la tabla 5, una gran mayoría de los informantes se dedicaba profesionalmente a labores agrícolas (jornalero, labrador, marinero, payés, pastor, etc.); por el contrario, hay muy pocos informantes ocupados en tareas no agrícolas y, la mayor parte, son oficios artesanos (carpintero, zapatero, herrero, etc.).

\begin{tabular}{|c|c|c|c|}
\hline \multirow{2}{*}{ Ocupación } & Agrícola & No agrícola & Total \\
\cline { 2 - 4 } & 165 & 31 & 196 \\
\hline
\end{tabular}

Tabla 5. Ocupación de los informantes del ALPI.

Por último, los datos informan sobre el grado de alfabetización de los informantes. Como se puede ver en la tabla, la mayor parte de los sujetos estaban alfabetizados, aunque sea en un grado elemental. Solo una cuarta parte de ellos eran analfabetos, contra tres cuartas partes que sabían leer, poco o mucho.

\begin{tabular}{|l|l|l|l|l|l|}
\cline { 2 - 3 } \multicolumn{2}{|l|}{ Sí } & No & Total \\
\hline \multirow{2}{*}{ ¿Sabe leer? ${ }^{27}$} & Sí & 102 & & & \\
\cline { 2 - 3 } & Un poco & 34 & \multirow{2}{*}{144} & 51 & 195 \\
\cline { 2 - 5 } Moco $^{29}$ & 8 & & & \\
\hline
\end{tabular}

Tabla 6. Alfabetización de los informantes del ALPI.

A propósito de la alfabetización de los informantes, el $A L P I$ ofrece unos datos muy interesantes desde el punto de vista de la sociolingüística histórica. Se trata de apreciaciones marginales sobre el grado de conocimiento del castellano (o del francés en la Cataluña del Norte) que aparecen en algunos cuadernos. Marginales porque no tenían ningún espacio reservado a ellas los cuestionarios, pero sin embargo importantes, puesto que gran parte de la encuesta se realizaba, si se podía, a partir de la traducción.

\footnotetext{
${ }^{27}$ Sin datos de tres informantes de Anna (cuaderno I) ni de Formiguera, Taurinyà, Cabestany, Banyuls, Bell-Iloc d'Urgell, Bordils, Cornellà de Llobregat (dos informantes) y Sant Joan (Eivissa) (cuadernos II).

${ }^{28}$ Se han unificado "un poco" y "algo".

${ }^{29}$ Se han unificado "muy poco" y "miserablemente".
} 


\begin{tabular}{|l|l|l|l|l|l|l|l|l|}
\hline \multirow{2}{*}{$\begin{array}{l}\text { ¿Habla } \\
\text { castellano? }\end{array}$} & Es bilingüe & Sí & Bastante & $\begin{array}{c}\text { Un } \\
\text { poco }^{30}\end{array}$ & $\begin{array}{c}\text { Muy } \\
\text { poco }^{31}\end{array}$ & $\begin{array}{c}\text { Lo entiende, } \\
\text { pero no lo habla }\end{array}$ & No & Total \\
\cline { 2 - 9 } & 3 & 18 & 3 & 28 & 9 & 12 & 29 & 102 \\
\hline
\end{tabular}

Tabla 7. Conocimiento del castellano de los informantes catalanes del ALPI.

En suma, los perfiles del $A L P I$ se corresponden mayormente con la voluntad explícita de sus autores, se adecuan a los modelos tradicionales de raíz gilliéroniana y, a diferencia del $A L C$, son coherentes con el espíritu motivador y con los principios fundamentales de la geolingüística europea tradicional, que corresponden al modelo del informante llamado NORM, excepto en lo que concierne a la edad de los sujetos, que se encuentra mayormente entre la mediana edad. El salto entre el $A L C$ y el $A L P I$ es notable, sobre todo por lo que a metodología se refiere. Sin embargo, el hecho de que el $A L P I$ fuera de ámbito ibérico y no solo catalán, de que se retrasase su publicación, y la interrupción del $A L C$, dieron lugar a un nuevo proyecto de atlas catalán: el Atles lingüístic del domini català.

\section{El Atles lingüístic del domini català, dirigido por Joan Veny y Lídia Pons}

\subsection{El proyecto de creación de un Atlas lingüístic del domini català}

El Atles lingüístic del domini català $(A L D C)$ es, sin duda, el más ambicioso de los tres que se analizan en este trabajo por lo que se refiere al catalán: mientras que tanto el $A L C$ como el $A L P I$ encuestan unas cien localidades, el $A L D C$ se basa en 190 puntos de encuesta. En 1952, Germà Colón y su maestro Antoni M. Badia i Margarit idearon el atlas y presentaron sus objetivos principales en la revista Orbis (Badía \& Colón 1952), editada por el centro de dialectología de la Universidad Católica de Lovaina. En el congreso de filología románica de Barcelona de 1953 - y cuyas actas se publicaron en 1955- ampliaron la información (Badía \& Colón 1952, 1955; v. también Perea 2020b).

\footnotetext{
30 Se han unificado "un poco", "algo" y "medianamente".

${ }^{31}$ Se han unificado "muy poco", "bastante mal" y "con dificultad".
} 
En el planteamiento inicial fue concebido como una superación del $A L C$, que había quedado inacabado durante la guerra y cuyas encuestas ya tenían más de 30 años y eran, pues, datos obsoletos (Badía \& Colón 1952: 403; 1955: 655), y como un complemento del $A L P I$, atlas que se esperaba con mucha expectación. De hecho, se planteaba como metodológicamente diverso, y más preciso - puesto que la extensión era más reducida y se podía cubrir un mayor número de puntos de encuesta.

Entre las muchas innovaciones metodológicas que el $A L D C$ proponía cabe centrarse en los perfiles de los informantes. Para la encuesta del ALDC Badia y Colón preveían tres cuestionarios: un cuestionario "normal", para la encuesta de todas las localidades; uno de "ampliado", cuyas respuestas no tenían que ser cartografiadas, y otro de "reducido", aplicable solo a 15 localidades. Los sujetos del cuestionario "normal" tenían que reunir parcialmente las características NORM: tenía que ser un único sujeto, hombre, de entre 35 y 50 años, agricultor - preferiblemente con una pequeña propiedad agrícola-, con una cierta inteligencia natural y sin mucha instrucción escolar (Badía \& Colón 1952: 405-406; 1955: 657-658). No se hacía comentario alguno sobre la ascendencia del mismo lugar, pero se supone que, siguiendo los parámetros de la geolingüística tradicional, fuese hijo del lugar y con poca movilidad. Para las 15 localidades del cuestionario "reducido" Badía y Colón proponían un modelo casi sociolingüístico; dicho cuestionario tenía que encuestarse a dos niños (12 y 15 años); dos jóvenes (20 y 28 años, es decir, antes del servicio militar y después de casarse); un adulto (el sujeto del cuestionario "normal") y un anciano (70 años); además, dichos perfiles de edad, pero femeninos, también se incluían en el proyecto. El cuestionario reducido, igualmente, preveía unas preguntas en la lengua oficial del estado (castellano, francés o italiano) para evaluar su conocimiento entre la población catalanohablante (Badía \& Colón 1952: 406; 1955: 656). En 1962 (BadiaMargarit et al. 1962) propusieron adaptar el modelo de cuestionario por imágenes (Pictorial Linguistic Interview Manual) que Stanley M. Sapon había presentado poco antes, hecho que permitía trabajar con tres sujetos a la vez; finalmente no prosperó dicho método en el ALDC, y se volvió al cuestionario tradicional. 
4.2 La realización del Atles lingüístic del domini català

Si el proyecto lo propusieron Antoni M. Badia y Germà Colón, este último no participó activamente en las encuestas y en la realización del atlas; en su lugar, Joan Veny tomó la dirección del proyecto al lado de Badia. Las encuestas se llevaron a cabo entre 1964 y 1978, y los materiales se publicaron entre 1989 y 2018, bajo la dirección de Joan Veny y de Lídia Pons. Aunque el nombre se mantuvo, hubo algunos cambios metodológicos importantes entre la presentación del proyecto y el inicio de las encuestas. Los primeros cambios tuvieron lugar ya antes del inicio del trabajo de campo, puesto que se abandonó la idea de contar con tres cuestionarios y se elaboró un único modelo con aspectos relacionados con la vida rural, "aplicable sobretot a camperols d'edat madura i avançada" (Veny \& Pons 1998: 11). En esta afirmación se ve, ya, el otro gran cambio, que tuvo lugar en el perfil de los informantes.

En la introducción digital del $A L D C$, se explica el modelo de informante que, teóricamente, seleccionaron para las encuestas: ${ }^{32}$

Els requisits ideals de l'informant eren edat avançada, intel-ligència natural, fill del poble, dentició acceptable, instrucció elemental o nul.la, sedentari, ascendència fixada a la localitat, cònjuge del mateix poble, familiarització amb les feines agrícoles i amb les activitats de la vida patrimonial de la població. En general, s'ha procurat que tant els informants com els seus pares fossin nadius de la població estudiada i, pel que fa al nivell d'instrucció, com que calia evitar individus amb un parlar sotmès a la interferència d'una altra llengua (castellà, francès, italià), s'ha recorregut a parlants poc escolaritzats. ${ }^{33}$

Como se ve, adoptan todos los del modelo NORM, incluso el principio de "edad avanzada", rompiendo así con la propuesta metodológica inicial de Badia y Colón. Se rompió, también, con la propuesta metodológica inicial por lo que se refiere al número de sujetos. Si en las propuestas de la década de 1950 se proponía seguir el principio

\footnotetext{
32 Parafraseado, pero, en esencia, con el mismo contenido, también en la edición en papel (ALDC, I: 10).

${ }^{33}$ https://aldc.espais.iec.cat/metodologia-2/preambul-2/informants/ [consulta: 18.ı.2021].
} 
del sujeto único, durante los trabajos de campo se encuestaron múltiples sujetos. Los directores del trabajo lo justifican de la siguiente forma:

Davant les dificultats de comptar durant tres o quatre dies amb els serveis d'un camperol, perquè el seu treball - llaurar un camp, dallar l'herba, munyir les vaques, etc. - és especialment actiu a l'època estival -que és quan es realitzava generalment l'enquesta-, es va decidir de recórrer a pluralitat d'informadors. En general, moltes parts de l'enquesta es van fer amb una persona acompanyada sovint del seu cònjuge o d'un amic; però també s'ha treballat amb un grup de tres o quatre subjectes, $\mathrm{i}$ hi ha enquestes amb un sol informador. ${ }^{34}$

En total, según indican los directores de la obra, se han encuestado 473 sujetos, pero solo se dan datos de 466, y no siempre completos, como es habitual en obras de estas características. Recientemente han aparecido en un volumen las crónicas del trabajo de campo en el que se puede reseguir la casuística que llevó a elegir a más o menos informantes en cada localidad (Veny \& Pons 2020).

\subsection{Perfiles sociolingüísticos de los informantes del ALDC}

En los materiales introductorios al atlas, los autores dan información sobre los sujetos encuestados. De los tres atlas, el $A L D C$ es el que ofrece datos más detallados sobre cada uno de ellos, aunque se eche en falta información sobre el grado de bilingüización de los sujetos, como puede extraerse del ALPI (cfr. §3) y como pretendían recoger Badia y Colón en el cuestionario reducido (§ 4.1). En concreto, informan del sexo, de la edad, de la naturaleza o no del lugar, de la profesión, del grado de instrucción, de la filiación de los padres y del cónyuge, y de las estancias fuera del lugar que hubiera podido hacer. Además, las ya mencionadas crónicas dan información extra que, en algunos casos, puede ser valiosa.

\footnotetext{
${ }^{34}$ https://aldc.espais.iec.cat/metodologia-2/preambul-2/informants/ [consulta: 18.ı.2021].
} 
De las 190 localidades encuestadas, ${ }^{35}$ hay un único sujeto en 38 de ellas (un $20 \%$ del total). La mayor parte del $A L D C$ fue encuestado, pues, con sujeto múltiple, como señalan sus directores. Generalmente fueron dos, tres o cuatro sujetos, pero en algunos casos extremos se llegó a los seis sujetos; en el cuestionario que se publicó inicialmente en 1965 hay espacio reservado para cuatro sujetos, así que hay que presuponer este es el número máximo que preveían de un modo normal (Badia i Margarit \& Veny i Clar 1965: 5-6).

Como se ve en la tabla siguiente, lo más habitual es que haya dos o tres sujetos; los casos de más de cuatro son ya muy raros. Algunos son explicables porque la encuesta se realizó en dos o más etapas, y el sujeto no pudo ser el mismo (son los casos de Sant Salvador de Guardiola, Tordera y Sóller).

\begin{tabular}{|l|l|l|l|l|l|l|l|}
\hline $\begin{array}{l}\text { Número de } \\
\text { informantes }\end{array}$ & 1 & 2 & 3 & 4 & 5 & 6 & Total \\
\cline { 2 - 8 } & 38 & 70 & 51 & 19 & 7 & 4 & 189 \\
\hline
\end{tabular}

Tabla 8. Número de informantes encuestados en el ALDC.

A parte del número de sujetos, los parámetros que se evalúan son el sexo, la edad, la formación, la profesión y la movilidad, así como la ascendencia en el municipio. ${ }^{36}$ En cuanto al sexo, se observa una presencia mayoritaria de varones, pero el papel de las mujeres no es para nada menospreciable. De 466 sujetos, 408 son varones, y 58, mujeres, de las que 14 aparecen como primer sujeto. Hay que destacar que hay dos localidades en las que solo fueron encuestadas mujeres, Llobera (Solsonés, Lérida) (dos sujetos) y Barcelona - barrio de Sants ${ }^{37}$ (un sujeto).

En la edad de los sujetos hay bastante disparidad, con una clara preferencia por los sujetos más ancianos, especialmente de la generación de los 70 años. Hay, incluso, cuatro sujetos de más de 90 años. Sin embargo, se observa como, efectivamente, se

\footnotetext{
${ }^{35}$ De Vall-de-roures no hay filiación de los sujetos; así, pues, hay datos de 189 localidades.

${ }^{36}$ Al final de la ficha técnica sobre las localidades y sus informantes, en la versión en papel del ALDC (I: 98-99) hay unos gráficos meramente descriptivos con algunas cuestiones que aquí se abordan: se describen las franjas de edad, los sexos y la instrucción - a parte de los en que fueron hechas las encuestas y el número de grabaciones magnetofónicas de los cuestionarios y los etnotextos. Esta información no está disponible en la versión web y, en cualquier caso, no difiere de los datos de este artículo.

${ }^{37}$ En Barcelona y Valencia se encuestó a un sujeto característico de un barrio con personalidad propia, que fuese representativo de este, no de toda la ciudad - no así en Palma.
} 
abandonó la propuesta inicial de encuestar a hombres de mediana edad y ganó la generación anciana. En cierto modo, esto hace que los datos del $A L P I$ y los del $A L D C$ sean fácilmente comparables, pues corresponden en general a individuos de la misma generación. $^{38}$

\begin{tabular}{|l|l|l|l|l|l|l|l|l|}
\hline \multirow{2}{*}{ Edad } & hasta 30 & $31-40$ & $41-50$ & $51-60$ & $61-70$ & $71-80$ & 81 o más & Total \\
\cline { 2 - 8 } & 4 & 8 & 27 & 51 & 147 & 170 & 59 & 466 \\
\hline
\end{tabular}

Tabla 9. Edad de los informantes del ALDC.

La condición de NORM se manifiesta, también, en la formación. Si bien en la década de 1960 y 1970 ya debía de ser muy difícil encontrar individuos analfabetos en todas las localidades, la mayor parte de ellos solo recibió instrucción primaria. ${ }^{39}$ Hay algunos sujetos, muy pocos, con instrucción secundaria y, excepcionalmente, algún caso de educación superior. De hecho, se trata solo de dos estudiantes tomados como segundo y tercer sujeto, respectivamente (en Prats de Balaguer y en Albalat de la Ribera), y del sacerdote Antoni Nughes, informante de la ciudad sarda de l'Alguer. Es una lástima - cabe insistir en ello- que no se tengan datos sobre el conocimiento de español, tanto entre los analfabetos como entre quienes tienen una educación primaria - algunos de los cuales quizá habían estudiado antes de la Guerra Civil, aún en catalán.

\begin{tabular}{|l|l|l|l|l|l|}
\hline \multirow{2}{*}{ Formación } & Analfabeto & Primaria & Secundaria & Estudios superiores & Total $^{40}$ \\
\cline { 2 - 6 } & 55 & 384 & 12 & 3 & 454 \\
\hline
\end{tabular}

Tabla 10. Nivel de formación de los informantes del ALDC.

\footnotetext{
${ }^{38}$ Sirva esta anécdota de ejemplo: en la crónica de la encuesta de l'Ametlla de Mar, de 1969, Lídia Pons escribe que el mismo sujeto de su encuesta, en 1940, fue informante de Sanchis y de Moll en las tareas del ALPI; Pons se queja de que el hombre se hace ya el sabio, pues anteriormente ha colaborado en una encuesta dialectal (Veny \& Pons 2020: 177).

${ }^{39}$ En el análisis se han omitido los años que tenía el sujeto cuando dejó la escuela, que en el caso de la instrucción primaria oscilan entre los 8 y los 12 .

${ }^{40}$ Hay algunos informantes de los que no se tienen indicaciones sobre formación; el informante de Mahón del que solo se dice "si" se ha asimilado a "instrucción primaria".
} 
$Y$ el otro gran factor que discriminaba tradicionalmente a los sujetos era su ocupación. Como se ha visto, tanto el proyecto inicial del ALDC de los años 50 como su desarrollo posterior en los 60 y 70 orientaban la elección del informante entre aquellos que se dedicaban, preferentemente, a la agricultura, puesto que una parte muy importante del cuestionario incluía palabras y conceptos propios de la vida rural. Así, no es de extrañar que la mayoría de los informantes tengan ocupaciones referidas al sector primario (agricultor, labrador, payés, pastor, marinero, vaquero, cazador, etc.). Hay que destacar, también, el gran número de amas de casa, la mayor parte de las cuales, sin embargo, puede incluirse entre el sector primario, puesto que son las esposas de los payeses, labradores y pastores que actúan de informantes principales. Finalmente, se consideran a parte los alguaciles municipales - a menudo combinando esta ocupación con otra agrícola-, hecho que se explica por el método que los investigadores seguían para elegir a un sujeto: llegados a la localidad, se dirigían al Ayuntamiento, donde el alcalde les indicaba posibles sujetos. Si el alguacil, ya disponible, era óptimo, se empezaba con él la encuesta (Veny \& Pons 2020).

\begin{tabular}{|l|l|l|l|l|l|l|}
\hline \multirow{2}{*}{ Ocupación } & $\begin{array}{l}\text { Agrícola, } \\
\text { marinera, rural }\end{array}$ & No agrícola ${ }^{41}$ & Alguacil & Ama de casa & Intelectual $^{42}$ & Total $^{43}$ \\
\cline { 2 - 7 } & 334 & 78 & 7 & 38 & 4 & 461 \\
\hline
\end{tabular}

Tabla 11. Ocupación de los informantes del ALDC.

Y, por último, hay las cuestiones de la movilidad de los sujetos y la ascendencia fijada en el lugar, además del hecho, casi absoluto, de que los informantes sean naturales del lugar. De hecho, solo 38 informantes (de un total de 466) no son naturales del lugar. En la mayor parte de ellos responde a dos casuísticas: o bien se trasladaron al lugar en su infancia o juventud, o bien son cónyuges de un informante que sí que es natural del lugar, como puede aclararse consultando las crónicas del trabajo de campo (Veny \& Pons 2020). Igualmente, solo 69 de los informantes no

\footnotetext{
${ }^{41}$ En esta categoría se han incluido tenderos (carniceros, horneros, etc.), hosteleros, sastres, modistas, mineros, transportistas, comerciantes en general, etc.

42 Sacerdotes, estudiantes y oficinistas.

${ }^{43}$ La suma total no da 466 porque de algunos informantes no se tienen datos sobre la profesión; además, hay que tener en cuenta que cuatro "alguacil" se computan también en "agrícola", puesto que desempeñan ambas ocupaciones.
} 
tienen ascendencia en el lugar de encuesta; si se descuentan aquellos informantes que no son naturales del lugar, se ve que solo 31 de los informantes "tradicionales", es decir, del lugar, tienen ascendencia totalmente foránea. Finalmente, 283 informantes han estado períodos largos fuera de la localidad, a menudo por motivos laborales o por prestar el servicio militar. ${ }^{44}$

\begin{tabular}{|c|c|c|c|c|}
\hline \multirow{2}{*}{$\begin{array}{c}\text { Movilidad } \\
\text { de los } \\
\text { informantes }\end{array}$} & $\begin{array}{c}\text { No son } \\
\text { naturales del } \\
\text { lugar }\end{array}$ & $\begin{array}{c}\text { Sin ascendencia fijada } \\
\text { en el lugar }\end{array}$ & $\begin{array}{c}\text { Más de 6 meses } \\
\text { fuera del lugar }\end{array}$ & Total \\
\cline { 2 - 3 } & 38 & $\begin{array}{c}69 \text { (incluyendo los } \\
\text { informantes que no son } \\
\text { naturales del lugar) }\end{array}$ & 283 & 352 \\
\cline { 2 - 3 } & & &
\end{tabular}

Tabla 12. Movilidad de los informantes del ALDC.

En resumen, el ALDC es el atlas más ambicioso del dominio catalán, también metodológicamente, es el que incluye más localidades y es lógico que sea también el que cuente con un mayor número de sujetos. Si bien en sus inicios los impulsores del ALDC idearon un atlas cuyos informantes no fuesen totalmente un modelo NORM, el desarrollo de las encuestas cambió esta tendencia y la mayor parte de los sujetos que participaron en los trabajos corresponden, finalmente, al modelo clásico de la geolingüística.

\section{Recapitulación: el valor desigual de los atlas catalanes}

En 1917 Alcover consideraba necesario encuestar, de cada localidad, al menos un representante de cada generación: de la joven, de la mediana, y de la anciana. Este planteamiento, ausente en el $A L P I$, reapareció bajo el cuestionario reducido en los planes de Badia y de Colón. Por desgracia, ni Griera quiso llevar a cabo el planteamiento de Alcover, ni los ejecutores del ALDC pudieron desarrollarlo como

\footnotetext{
${ }^{44}$ Se ha considerado una estancia "larga" a partir de los seis meses de ausencia. No se han tenido en cuenta, pues, estancias breves de semanas, a menudo en pueblos de la misma comarca o provincia, que los investigadores del ALDC consignan pero que no se consideran relevantes.
} 
imaginaron Badia y Colón en sus orígenes. El resultado es que todos los atlas de que se dispone ofrecen una visión necesariamente parcial de la lengua siguiendo los parámetros de la dialectología tradicional.

Los tres atlas que se han considerado en este trabajo - el $A L C$, el $A L P I$ y el $A L D C$ - son obras muy desiguales entre sí. Mientras que el $A L C$ apenas tiene valor lingüístico $\mathrm{y}$, hoy en día, se valora solo desde el punto de vista histórico o historiográfico, el $A L D C$ se ha convertido, una vez completada su publicación, en el atlas de referencia para la catalanofonía, una fotografía muy valiosa del catalán de las décadas 1960 y 1970. El ALPI, que podría ser un complemento fantástico al ALDC, como punto de comparación con la generación casi inmediatamente anterior a la del $A L D C$, aguarda paciente su publicación. Son tres atlas, en efecto, con un valor lingüístico muy desigual.

Este valor desigual también se observa en el enfoque metodológico de cada trabajo, y de un modo muy especial en la selección de las localidades y de los informantes, quizá los dos elementos más importantes de cualquier proyecto de cartografía lingüística. El $A L C$ es, sin duda, un trabajo no solo obsoleto, sinó absolutamente inválido desde sus inicios desde el punto de vista metodológico. No solo porque a partir del volumen VI los informantes sean 40 años más jóvenes, y la realidad (socio)lingüística del catalán de 1920 no sea la de 1959, sino que ya en la elección de los informantes originales se observaban graves problemas. Como se ha visto, Griera no respetó el principio de unidad generacional en su atlas, y se contradijo a si mismo tomando una cantidad abrumadora de informantes jóvenes. Son conocidas - y merecidas - las críticas a Griera también por elegir sujetos "intelectuales", es decir, personas con formación académica, que sin duda modifican la espontaneidad y la "genuinidad" (sea lo que quiera que signifique esta expresión) que se buscaba en las labores dialectológicas tradicionales. Con el agravante, claro, de que tomó eminentes lingüistas como Fabra, Barnils o Fullana como sujetos válidos-e incluso él mismo se tomó como informante de su pueblo. Quizá solo el principio de utilizar hombres, y no mujeres, como informantes, se respeta de un modo sistemático y eficaz.

Si el $A L C$ es casi un contramodelo a la metodología tradicional, el $A L P I$ es, en cambio, un alumno aventajado. Cumple con casi todos los criterios de la geolingüística 
tradicional resumidos en el acrónimo NORM: sus informantes son mayormente varones, rurales, de localidades pequeñas, de ocupaciones agrícolas, con muy poca o nula formación académica y, a menudo, monolingües. El único aspecto en que el $A L P I$ discrepa del patrón NORM es en la edad de los sujetos: la mayor parte son de mediana edad, y no ancianos. Además, es el único atlas que da informaciones acerca del grado de bilingüización de los sujetos, unos datos sin duda interesantes que tendrían que estudiarse con más detalle. Quizá el único reparo que puede $-y$ debe- hacerse al $A L P I$ es la rotura de la unidad generacional a causa del golpe que supuso la guerra civil. En efecto, las encuestas iniciadas en 1934 acabaron en 1952; no solo es importante de este corte los 18 años que separan las primeras encuestas de las últimas, sino todos los cambios sociopolíticos, demográficos $-y$, por lo tanto, sociolingüísticos- que supuso la guerra civil y los primeros años del franquismo. Sin embargo, ya en la reseña que Colón y Lütdke dedicaron al primer volumen del $A L P I$, aseguran que de este tipo de objeciones "no cabe acusar solo al ALPI", puesto que otros atlas románicos tampoco habían sido muy exigentes en la cuestión de la unidad generacional, sin que hubiese guerra civil de por medio (Colón \& Lütdke 1965: 325-326).

Este único problema "grave" del $A L P I$ tenía que remediarse con el $A L D C$, al menos en la parte catalana - que es en la que se centra este trabajo. Este nuevo proyecto de atlas, mucho más ambicioso en sus orígenes, tampoco consiguió realizar las encuestas en un lapso corto de tiempo: se hicieron entre 1964 y 1977 . Sin embargo, es sin duda un muy buen atlas, con materiales utilísimos para la geolingüística catalana y, como ya dijo Colón, "una obra que [...] podem considerar de les millors que, en el camp de la geografia lingüística, s'hagin mai realitzat en el conjunt dels països de Ilengua romànica" (2011: 357). Desde el punto de vista metodológico, sigue de cerca el modelo de informante NORM, y, si bien es frecuente encontrar sujetos que no lo cumplan, también lo es que el $A L D C$ encuesta el doble de poblaciones que el $A L C$ y el $A L P I$, y además con más de un sujeto. El resultado es que, si el $A L C$ se realizó con apenas 100 informantes, y el ALPI con casi 200, el ALDC cuenta con más de 450: era inevitable, pues, que no se tomasen todos los informantes tan prototípicamente NORMs. 
En síntesis, de los tres atlas lingüísticos completos de los que dispone el catalán, solo el $A L D C$ toma informantes que encajan en los parámetros clásicos del NORM. Sin embargo, junto al $A L D C$ hay que considerar el $A L P I$, cuya publicación permitirá de tener un material lingüístico de un impagable valor histórico que podrá, a su vez, ser comparado con los datos del $A L D C$, mucho más completos y ahora también ya dotados de un innegable valor diacrónico. Por el contrario, el $A L C$ tiene un valor historiográfico indiscutible, puesto que fue pionero fuera del ámbito francés, pero su deficiente metodología lo invalida para aprovechar sus materiales lingüísticos.

\section{Referencias}

Alcover, Antoni M. (2020) Obres completes V: Manifests, Maria-Pilar Perea (ed.), Palma: Nova Editorial Moll.

BADIA I MARgARIT, Antoni \& Joan Veny I CLAR (1965) Atlas lingüístic del domini català: Qüestionari, Barcelona: Vda. Fidel Rodríguez Ferrán.

Badia-Margarit, Antoni; Germà Colón; Manuel Companys \& Joan Veny Clar (1962) "Atlas lingüístic del domini català: Estado de los trabajos. Aspectos metodológicos", Boletim de Filologia. Actas do IX Congreso Internacional de Linguística Românica, III, 121-126.

BADíA, Antoni \& Germà CoLón (1952) "Atlas linguistique du domaine catalan", Orbis, 1, $403-$ 409.

BADíA, Antoni \& Germà Colón (1955) "L'Atlas lingüístic del domini català", in Antoni Badía, Antoni Griera \& Frederic Udina (eds.), VII Congreso Internacional de Lingüística Románica. Tomo II. Actas y memorias, Barcelona: Abadía de Sant Cugat del Vallés, 655-660.

Chambers, J. K \& Peter Trudgill (1998) Dialectology, Cambridge: Cambridge University Press, 2ª edición.

COLóN, Germà (1961) "Autour de l'atlas linguistique d'Andorre", Zeitschrift für romanische Philologie, 77(1-2), 49-69.

COLóN, Germà (1997) "Entorn de l'Atlas lingüístic d'Andorra", in Estudis de filologia catalana i romànica, València/Barcelona: Institut Interuniversitari de Filologia Valenciana/Publicacions de l'Abadia de Montserrat, 305-327. 
Colón, Germà (2011) "L'Atles lingüístic del domini català", in Lexicografia, lèxic i crítica textual, Castelló/Barcelona: Fundació Germà Colón/Publicacions de I'Abadia de Montserrat, 357360.

ColóN, Germà \& Helmut LÜDTKE (1965) "[Reseña al] Atlas lingüístico de la Península Ibérica", Vox Romanica, 24, 323-335.

Cortés CARRERES, Santi \& Vicent García Perales (2009) La historia interna del Atlas lingüístico de la Península Ibérica ( $A L P I)$, València: Publicacions de la Universitat de València.

GARCía Mouton, Pilar (2020) "Las mujeres como sujetos de encuesta en el Atlas lingüístico de la Península Ibérica (ALPI)", in M.a José Martínez Alcalde; Juan Pedro Sánchez Méndez; Francisco Javier Satorre Grau; Mercedes Quilis Merín; Amparo Ricós Vidal; Adela García Valle; Francisco Pedro Pla \& Santiago Vicente Llavata (ed.), El español y las lenguas peninsulares en su diacronía: miradas sobre una historia compartida. Estudios dedicados a M. $\underline{a}$ Teresa Echenique Elizondo, València: Tirant Humanidades/Université de Neuchâtel, 209-228.

GARGALlO, José Enrique (1990) “Aspectos metodológicos en la elaboración del ALDC («Atlas Lingüístic del Domini Català»). Valoración y cotejo con otros atlas previos", Revista de Filología Románica, 7, 175-190.

GrierA, Antoni (1923) "Introducció", in Atlas lingüístic de Catalunya, Barcelona: Polígraf, [s. p.]. GRIERA, Antoni (1963) Memòries, Sant Cugat del Vallès: Instituto Internacional de Cultura Románica.

GRIERA, Antoni (1964) Atlas lingüístic de Catalunya: Introducció explicativa, Sant Cugat del Vallès: Instituto Internacional de Cultura Románica.

GonzÁlez GonZÁlez, Manuel (1992) “Metodología de los Atlas lingüísticos en España”, in Nazioarteko Dialektologia Biltzarra. Agiriak, Bilbao: Euskaltzaindia, 151-177.

JULIÀ-MUNÉ, Joan (2019) Un segle de lingüística catalana: de la Lletra de convit a la GCC (19012002), Lleida: Edicions de la Universitat de Lleida.

MichelenA, Luis (1960) "[Reseña de] A. Griera, Vocabulario vasco. (Ensayo de una interpretación de la lengua vasca)", Instituto Internacional de Cultura Románica, Abadía de San Cugat del Vallés, 1960", Boletín de la Sociedad Vascongada de Amigos del País, 16(3), 384-387.

MiCHELENA, Luis (1985) "Vocabulario vasco (Ensayo de una interpretación de la lengua vasca)", in Lengua e historia. Madrid: Paraninfo, 329-333. 
NAVARRO TOMÁs, Tomás (1975) "Noticia histórica del ALPI" in Capítulos de geografía lingüística de la Península Ibérica, Bogotá: Instituto Caro y Cuervo, 9-21.

PereA, Maria-Pilar (2008) Epistolari d'Antoni M. Alcover (1880-1931), Palma: Govern de les Illes Balears/Editorial Moll [recurso en CD-ROM].

PereA, Maria-Pilar (2020a) "Els manifests d'Antoni M. Alcover (1917-1924)", in Antoni M. Alcover, Obres completes V: Manifests, Maria-Pilar Perea (ed.), Palma: Nova Editorial Moll, 7-30.

PEREA, Maria-Pilar (2020b) “El reflex d'alguns trets fonètics i morfològics valencians en l'ALPI i en l'ALDC", Itaca. Revista de Filologia, 11, 33-72.

SANCHIS GUARNER, Manuel (1953) La cartografía lingüística en la actualidad y el Atlas de la Península Ibérica, Madrid: Consejo Superior de Investigaciones Científicas.

SANCHIS GUARNER, Manuel (1955) "La cartografía lingüística catalana", in Antoni Badía, Antoni Griera \& Frederic Udina (eds.), VII Congreso Internacional de Lingüística Románica. Tomo II. Actas y memorias, Barcelona: Abadía de Sant Cugat del Vallés, 647-654.

SANChis Guarner, Manuel; Lorenzo Rodríguez-CaStellano; Aníbal Otero \& Luis L. Lindley CinTRA (1962) "El Atlas lingüístico de la Península Ibérica (ALPI). Trabajos, problemas y métodos", Boletim de Filologia. Actas do IX Congreso Internacional de Linguística Românica, III, 113120.

VENY, Joan (1986) Introducció a la dialectologia catalana, Barcelona: Enciclopèdia Catalana.

VENY, Joan \& Lídia PONS (1998) Atles lingüístic del domini català: Qüestionari, Barcelona: Institut d'Estudis Catalans.

VenY, Joan \& Lídia Pons (2020) (ed.) Atles lingüístic del domini català: Cròniques del treball de camp, Barcelona: Institut d'Estudis Catalans.

Atlas lingüísticos

AIS = Karl Jaberg \& Jakob Jud (1928-1940) Sprach- und Sachatlas Italiens und der Südschweiz / Atlante linguistico ed etnografico dell'Italia e della Svizzera meridionale, 8 vols., Zofingen: Ringier. [también en línea en: https://www3.pd.istc.cnr.it/navigais-web/, consulta 18.I.2021].

ALA = Antoni Griera (1960) Atlas lingüístic d'Andorra, Barcelona: Polígraf.

ALC = Antoni Griera (1923-1964) Atlas lingüístic de Catalunya, 8 vols., Barcelona/Sant Cugat del Vallès: Políograf/Instituto Internacional de Cultura Románica. 
Dialectologia. Special issue, IX (2021), 7-37.

ISSN: 2013-2247

ALDC = Joan Veny \& Lídia Pons (dir.) (2001-2018) Atles lingüístic del domini català, 9 vols., Barcelona: Institut d’Estudis Catalans. [también en línea en https://aldc.espais.iec.cat, consulta 18.I.2021].

ALDT = Lluís Gimeno Betí (1997) Atles lingüístic de la Diòcesi de Tortosa, Barcelona: Institut d'Estudis Catalans.

ALM = Francesc de B. Moll (en línea) Atles lingüístic mariner, Palma: GRESIB: Universitat de les Illes Balears [https://gresib.uib.cat/Projectes-de-recerca/Variacio/Atles-linguistic/ consulta 18.I.2021].

ALPI = Tomás Navarro Tomás (dir.) (1962) Atlas lingüístico de la Península Ibérica, 1 vol., Madrid: Consejo Superior de Investigaciones Científicas

ALPO = Henri Guiter (1966) Atlas linguistique des Pyrénées Orientales, Paris: Centre National de la recherche scientifique.

ALTA = Pere Navarro (1996) Els parlars de la Terra Alta, 2: Atles lingüístic de la Terra Alta, Tarragona: Diputació de Tarragona.

PALDC = Joan Veny (2007 - ) Petit atles lingüístic del domin català, 7 vols. (+ 2 en preparación), Barcelona: Institut d’Estudis Catalans. 